\title{
Overcoming Barriers and Limitations - Why This New Journal Is Needed
}

\author{
Winfried Rief ${ }^{a}$, Cornelia Weise ${ }^{\mathrm{a}}$
}

[a] Division of Clinical Psychology and Psychotherapy, Department of Psychology, Philipps-University of Marburg, Marburg, Germany.

Clinical Psychology in Europe, 2019, Vol. 1(1), Article e32600, https://doi.org/10.32872/cpe.v1i1.32600

Published (VoR): 2019-03-29

Corresponding Author: Cornelia Weise, Division of Clinical Psychology and Psychotherapy, Department of Psychology, Philipps-University of Marburg, Marburg, Germany. E-mail: weise@uni-marburg.de

\section{Highlights}

- Introducing the new journal Clinical Psychology in Europe (CPE).

- Overcoming artificial barriers by focusing on evidence instead of traditions.

- Bridging the gap from basic experimental to treatment-related research.

- Supporting open science recommendations.

- Covering a broad variety of research efforts.

- Full open access but no publication fees.

We warmly welcome you to the reading of our newly founded journal Clinical Psychology in Europe - CPE!

Most of us receive requests to submit a manuscript to some obscure new journal just about every day. And today you are holding another new journal in your hands and may - with good reason - be wondering whether it is really necessary to launch a new journal given the numerous existing options for submissions.

Our resounding answer is: Yes, we need this journal "Clinical Psychology in Europe" (CPE). Not because we feel the need to add another obscure new journal to the field, but because we are keen to have a journal that is committed to encouraging a modern and self-critical discussion in the scientific community, to have a journal that is open-minded about topics considered for publication, to have a journal that increases the visibility of our field of research and to have a journal that provides innovative ideas for future research in clinical psychology. 
CPE aims to face several challenges in the field of clinical psychology:

A first challenge stems from the past, when clinical psychology limited its power and potential to influence society with tensions and artificial barriers between traditions, especially between traditions of psychotherapy ("schools").

To face this challenge, CPE is clearly committed to evidence-based treatments, independent of their traditional roots. We encourage all innovations that attempt to overcome these kinds of barriers, we aim to integrate different approaches, and to find a common language in clinical psychology. Although science thrives on critical debates, we should aim to have these constructive debates inside our community - while acting as one powerful group for societal aims.

Secondly, it is all too often forgotten that clinical psychology is not limited to treatment. The fruitful exchange between basic approaches, mechanistic and experimental research, diagnostics and classification, epidemiology and interventional research is the basic motor of our science. Interventions without links to basic sciences are isolated applications, but not serious fields of research. Relating the different fields of clinical psychology to each other, but also with the progress of other areas such as neuroscience, emotion regulation, learning, social interaction, and many others, creates the cross-links that characterize top scientific fields.

Accordingly, in CPE we aim to provide a balanced ratio of articles reporting on basic, mechanistic, and experimental research in clinical psychology, research from associated areas, such as neuroscience, behavioral medicine, or health psychology and articles presenting treatment-related issues. Our goal is to stimulate interdisciplinary exchange and understanding.

A third critical challenge (not only for clinical psychology, but for science in general) is the risk of disseminating false positive results. Clinical psychology, and in particular intervention research, is particularly prone to this threat. Many psychotherapy researchers are strongly identified with their favorite approaches and theories, and sometimes tend to disrespect one major rule of "Good Research Practice": Distrust your own research, and cross-check every result critically before you attempt to publish it. This disrespect is problematic as the dissemination of false positive results misleads other researchers, deceives society, and leads to misallocation of resources.

To face this challenge, CPE supports open science endeavors. We do not consider current proposals for open science as the final result of these discussions, but as a process during which we should try and evaluate different approaches to continuously improve the validity of published results. Our supporting publisher "Leibniz Institute for Psychology Information (ZPID)" provides several tools to improve open science, for example literature databases, archives for data-sharing, repositories, support for study planning or pre-registration (https://www.leibniz-psychology.org/en/).

Fourth, it is our impression that there is extensive knowledge around, but this knowledge does not always reach the scientific community. Given the pressure to 'publish or 
perish', many researchers focus on submitting empirical research articles. Journals welcome these manuscripts and only rarely allow for updates or general overviews. Accordingly, there are limited opportunities for experts to share knowledge they have accumulated over several years of work in a specific field.

To reflect the broad variety of research efforts, CPE provides the opportunity to submit different types of articles. For empirical research, the typical Research Articles can be submitted. However, we also encourage submitting Scientific Updates on the current knowledge of a field in which experts can share their current summaries with all of us. The same holds true for the publication of other expertise or events, such as inaugural speeches or keynote lectures, which are characterized by thorough preparation. CPE can help to further disseminate this knowledge using the format of State-of-the-Art Overviews. To meet our goal of providing a platform of exchange, CPE further encourages authors to report Latest Developments (such as new technical applications or recently developed questionnaires) as well as topics related to Politics and Education in the field of clinical psychology. For example, the description of different legal regulations for clinical psychology and psychological interventions might inform and stimulate the development of such regulations in different countries.

And finally, we face a challenge in meeting the major societal aim of research: Disseminate it for the benefit of others and let others make use of it. This is why journals exist and why we meet at conferences. However, many journals have developed a life of their own with financial benefit becoming more and more relevant. As we are convinced that the decision about publication should not depend on whether authors can afford to pay substantial article processing charges we are delighted that - thanks to the support of Leibniz Institute for Psychology Information - our aim of not charging any publication costs for articles has become reality!

We welcome your submissions at https://cpe.psychopen.eu and we are looking forward to collaborating with you! Now enjoy reading the first issue of CPE and get inspired.

Winfried Rief, Editor in Chief, and Cornelia Weise, Managing Editor 